\title{
Atrial Flutter Versus Atrial Fibrillation in a General Population: Differences in Comorbidities Associated With Their Respective Onset
}

\author{
Ravi K. Mareedu, MD; Ihab B. Abdalrahman, MD; Kodlipet C. Dharmashankar, MD; \\ Juan F. Granada, MD; Po-Huang Chyou, PhD; Param P. Sharma, MD; Peter N. Smith, MD; \\ John J. Hayes, MD; Robert T. Greenlee, PhD and Humberto Vidaillet, MD
}

\begin{abstract}
Objective: Determine and compare the prevalence of known risk factors for cardiovascular disease among unselected individuals presenting with their first ever episode of atrial flutter (AFL) and atrial fibrillation (AF).

Study Design and Setting: We evaluated II pre-selected clinical variables including age, sex, smoking history and other potential cardiac risk factors. Using the resources of the Marshfield Epidemiologic Study Area, a population-based database, all newly diagnosed cases of either AFL or AF in the region during a 4-year period were identified.

Results: Among the 472 incident cases, 76 (16.1\%) had AFL and 396 (83.9\%) had AF. Compared to those with AF, subjects with AFL were more likely to have had a history of chronic obstructive pulmonary disease $(25 \%$ vs. $12 \%, P=0.006)$, heart failure $(28 \%$ vs. $17 \%, P=0.05)$, and smoking $(49 \%$ vs. $37 \%, P=0.06)$. Hypertension, on the other hand, was more common among individuals with $A F$ $(63 \%$ vs. $47 \%, P=0.01)$.

Conclusion: This study represents the first report to evaluate potential differences in the conditions associated with the development of AFL versus AF. Research into the mechanisms of atrial arrhythmogenesis may lead to improved preventive and therapeutic interventions.
\end{abstract}

Keywords: Arrhythmias, Clinical electrophysiology, Drugs, Epidemiology

\begin{abstract}
A Itrial flutter (AFL) and atrial fibrillation (AF) are the two most common sustained cardiac arrhythmias encountered in clinical practice. Recent advances in our understanding of the distinct electrophysiologic mechanisms responsible for AFL and AF have led to specific anatomically-based curative procedures. 1,2

Unlike other common cardiovascular disorders such as atherosclerosis, heart failure, and sudden death in which detailed knowledge about specific predisposing risk factors have resulted in effective preventive strategies and improved assessment of individuals at risk, the conditions responsible for AFL and AF, and the reasons why any given patient may develop one of these arrhythmias and not the other remain largely unknown.
\end{abstract}

\section{Methods}

Marshfield Epidemiologic Study Area (MESA)

MESA is a population-based cohort established in 1991 to support epidemiologic research within the context of integrated health care delivery. ${ }^{3}$ Such studies are possible in central Wisconsin because nearly all residents choose to receive their medical care from Marshfield Clinic (with its 40 regional centers) and St. Joseph's Hospital. The central portion of MESA includes 14 ZIP codes in Marshfield and surrounding communities, and is home to more than 54,000 residents. In this region, Marshfield Clinic's electronic medical record systems have been shown to capture $97 \%$ of the people, more than $90 \%$ of outpatient visits, $99 \%$ of deaths, and $95 \%$ of hospital stays. ${ }^{4}$ Residents of MESA represent a well-defined, unselected population, reducing the
Corresponding Author: Humberto Vidaillet, MD, Cardia Electrophysiology, Department of Cardiology, Marshfield Clinic, 1000 North Oak Avenue, Marshfield, WI 54449, Tel: 7I5-387-5080, Fax: 7I5-389-5757,

E-mail address: vidaillet.humberto@marshfieldclinic.org
Received: February 4, 2009

Revised: September 25, 2009

Accepted: October 7, 2009

doi: $|0.3121 / \mathrm{cmr} .2009 .85|$
Grant Support: Grant IULIRR0250II, Clinical and Translational Science Award (CTSA) program of the National Center for Research Resources, National Institutes of Health 
referral bias often influencing other health care study populations. With daily updates about the health care status and population characteristics, MESA provides a unique opportunity to conduct epidemiological association and frequency studies, and has supported a number of studies of cardiac arrhythmias and other cardiovascular conditions. ${ }^{4-9}$ This investigation was reviewed and approved by the Marshfield Clinic Research Foundation's Institutional Review Board.

\section{Electrocardiographic (ECG) Definitions}

ECG definitions have been detailed earlier, ${ }^{7}$ and they are summarized below. Only patients satisfying ECG documentation of their first ever episode of either AFL or AF were enrolled. A cardiac electrophysiologist confirmed all ECG diagnoses. While the minimum duration of continuous ECG documentation of each of these arrhythmias required for study was 5 seconds, virtually all incidents lasted minutes to days. ECG criteria used for study entry was as follows:

- AFL was considered to be present if there were visible and highly regular " $F$ " waves at a rate $\leq 350$ beats per minute. Highly regular "F" waves were defined as those in which the cycle to cycle atrial variability was $\leq 10 \mathrm{msec}$. Atrial rate in AFL had to be $>190$ beats per minute among patients receiving classes IA, IC and/or class III antiarrhythmic agents. In all others, the lowest acceptable atrial rate was 240 beats per minute.

- AF was defined by the presence of fibrillatory waves of variable size, shape and timing associated with an irregular ventricular response when atrioventricular response is intact.

\section{Patient Selection Criteria}

To identify potential incident cases of AFL or AF in MESA occurring from July 1, 1991 through June 30, 1995, we used
Marshfield Clinic's diagnostic database. Since 1979, this database has used the International Classification of Diseases $9^{\text {th }}$ Revision (ICD-9) to track all diagnoses recorded by Marshfield Clinic patients in the inpatient and outpatient setting. In addition to ICD 427.31 (AF) and 427.32 (AFL), we screened for potential cases using 7 additional diagnostic codes including 410 (acute myocardial infarction), 426.7 (Wolff-Parkinson-White syndrome), 427.0 (paroxysmal supraventricular tachycardia), 427.2 (paroxysmal tachycardia, unspecified), 427.81 (sinoatrial node dysfunction), 427.89 (other rhythm disorder, ectopic, nodal and wandering atrial pacemaker) and 427.9 (cardiac dysrhythmia, unspecified). While all potential cases were assessed for study entry, final inclusion required ECG confirmation by cardiac electrophysiologists. Given that the focus of this investigation was to evaluate the possible association of certain clinical variables with the initial onset of AFL or AF, subjects who in addition to their primary arrhythmia also had antecedent or concomitant AFL or AF, on detailed review of the medical records, were excluded. During the 220,000 person-years of observation, approximately 29,000 electrocardiograms and rhythm strips, 1100 Holter monitors and 500 ambulatory event recordings were obtained from MESA residents.

\section{Clinical Variables and Data Collection}

A written glossary defining all premorbid conditions under consideration was developed a priori. These included age, gender, heart failure, chronic pulmonary disease, systemic hypertension, previous stroke, myocardial infarction, rheumatic heart disease, smoking history, thyroid abnormality and diabetes mellitus. The full medical record of each incident case, including admission, discharge, inpatient and outpatient procedure, and clinical notes was abstracted by a trained study nurse to determine the presence of premorbid conditions prior to the diagnosis of the atrial arrhythmia. Quality assurance methods to ensure data integrity included

Table 1. Clinical characteristics present at the time of initial diagnosis of atrial flutter versus atrial fibrillation in a general population in the Marshfield Epidemiologic Study Area (MESA).

\begin{tabular}{lccc}
\hline Clinical characteristics & AFL $(\mathbf{n = 7 6 )}$ & AF (n=396) & $\boldsymbol{P}_{\text {-value* }}$ \\
\hline Chronic obstructive pulmonary disease & $19(25 \%)$ & $48(12 \%)$ & 0.006 \\
Hypertension & $36(47 \%)$ & $250(63 \%)$ & 0.01 \\
Congestive heart failure & $21(28 \%)$ & $69(17 \%)$ & 0.05 \\
Smoking history & $37(49 \%)$ & $146(37 \%)$ & 0.06 \\
Myocardial infarction & $17(22 \%)$ & $63(16 \%)$ & 0.18 \\
Diabetes mellitus & $12(16 \%)$ & $87(22 \%)$ & 0.28 \\
Thyroid disease & $9(12 \%)$ & $38(10 \%)$ & 0.53 \\
Previous stroke & $13(17 \%)$ & $62(16 \%)$ & 0.73 \\
Rheumatic heart disease & $1(1 \%)$ & $5(1 \%)$ & 1.00 \\
Male gender (\%) & $47(62 \%)$ & $210(53 \%)$ & 0.17 \\
Mean age, years (SD, range) & $70.0(12.4,25-95)$ & $72.0(12.5,21-94)$ & 0.22 \\
\hline
\end{tabular}

*Based on Fisher's exact test.

AFL, atrial flutter; AF, atrial fibrillation; SD, standard deviation. 
Table 2. Odds ratio* and $95 \%$ confidence interval for atrial flutter versus atrial fibrillation according to clinical characteristics.

\begin{tabular}{lccc}
\hline Clinical characteristics & OR & Cl & P-value \\
\hline Chronic obstructive pulmonary disease & 2.34 & $1.26,4.32$ & 0.007 \\
Hypertension & 0.56 & $0.34,0.93$ & 0.025 \\
Congestive heart failure & 2.00 & $1.12,3.58$ & 0.019 \\
Smoking history & 1.46 & $0.86,2.48$ & 0.163 \\
Myocardial infarction & 1.67 & $0.90,3.10$ & 0.103 \\
Diabetes mellitus & 0.66 & $0.34,1.28$ & 0.222 \\
Thyroid disease & 1.46 & $0.66,3.21$ & 0.352 \\
Previous stroke & 0.94 & $0.40,2.21$ & 0.143 \\
Rheumatic heart disease & 1.12 & $0.13,9.91$ & 0.144 \\
\hline
\end{tabular}

${ }^{*}$ Adjusted for age and gender.

OR, odds ratio; $\mathrm{Cl}$, confidence interval.

re-abstraction of every 10 th medical record, double data entry of a random sample of $10 \%$ of collected information, as well as range and edit checks on all collected data. All predetermined quality assurance parameters were exceeded.

\section{Statistical Analysis}

Clinical variables in the analysis included age, sex, congestive heart failure $(\mathrm{CHF})$, chronic obstructive pulmonary disease (COPD), hypertension, previous stroke, myocardial infarction, rheumatic heart disease, smoking history, thyroid abnormality, and diabetes mellitus. Fischer's exact test was performed to compare the difference (in percentage) between AFL patients and AF patients for each of the 11 above-mentioned clinical characteristics. A $P$-value of $<0.05$ was claimed statistically significant. Multivariate logistic regression analysis was performed to obtain odds ratio (OR) and corresponding 95\% confidence interval (CI) for the status of AFL for each of the preexisting comorbidities, with adjustment for age and gender. Additional analysis was done including the adjustment for other significantly different pre-existing comorbidities. All analyses were performed using SAS, version 9.1 (SAS Institute, Cary, North Carolina, United States).

\section{Results}

During the 4 years of ascertainment, a total of 472 MESA residents were found to have their first ever episode of AFL or AF. These included 76 (16.1\%) with AFL and 396 (83.9\%) with AF. We excluded 105 subjects who developed both rhythm abnormalities during the incidence period. Important differences were observed in 3 of the 11 variables pre-selected for analysis. Compared with those with AF, patients with AFL were more likely to have had a history of COPD (25\% vs. $12 \%, P=0.006)$, CHF (28\% vs. $17 \%, P=0.05)$, and a trend toward more smoking ( $49 \%$ vs. $37 \%, P=0.06$ ). Patients with $\mathrm{AF}$, on the other hand, were more likely to have had a history of hypertension (63\% vs. $47 \%, P=0.01)$.

As shown in table 1, there were no differences between the two patient groups with respect to their mean age, gender distribution, or likelihood of having had previous myocardial infarction, thyroid disease, rheumatic heart disease, previous stroke, or diabetes mellitus.

Although there was a non-significant difference in distribution of age (AFL 70.0 vs. AF 72.0, $P=0.22$ ) and males (AFL 62\% vs. AF $53 \%, P=0.17$ ) between both groups, we adjusted for both of them, as they could play a confounding role in other associations. After adjusting for age and gender, the associations identified in unadjusted analyses remained. Patients with AFL were twice as likely to have had a history of COPD (OR 2.34, 95\% CI: 1.26, 4.32, $P=0.007$ ), and CHF (OR 2.00, 95\% CI: 1.12, 3.58, $P=0.019$ ) compared to those with AF. Also, AFL patients were half as likely to have had a history of hypertension (OR 0.56, 95\% CI: 0.34, 0.93,

Table 3. Odds ratio* and 95\% confidence interval for atrial flutter versus atrial fibrillation according to relevant clinical characteristics.

\begin{tabular}{lccc}
\hline Clinical characteristics & OR & \multicolumn{1}{c}{ Cl } & \multicolumn{1}{c}{$\boldsymbol{P}$-value } \\
\hline Chronic obstructive pulmonary disease & 1.99 & $1.04,3.82$ & 0.038 \\
Hypertension & 0.51 & $0.30,0.86$ & 0.011 \\
Congestive heart failure & 1.87 & $1.00,3.50$ & 0.050 \\
Age & 0.99 & $0.97,1.01$ & 0.265 \\
Male & 1.15 & $0.66,1.98$ & 0.624 \\
\hline
\end{tabular}

${ }^{*}$ Adjusted for age, gender, and other clinical variables included in the same table.

OR, odds ratio; $\mathrm{Cl}$, confidence interval. 
$P=0.025)$ compared to AF patients. Differences in smoking distribution between the groups remained non-significant. There was no significant difference in other clinical characteristics between the two groups. Table 2 summarizes these results.

These statistically significant associations persisted even with COPD, CHF, and hypertension simultaneously included in a multivariate logistic regression model, in addition to age and gender as summarized in table 3 .

\section{Discussion}

$\mathrm{AF}$ and AFL are the two most common sustained cardiac arrhythmias encountered in clinical practice. $6,10-12$ While it is widely recognized that AF is much more common than AFL, neither the magnitude nor the reasons for this difference are well established. Our investigation evaluated potential differences in the conditions associated with the development of AFL versus AF.

Based on data derived from population-based studies, we have estimated that annually in the United States there are a total of approximately 200,000 incident cases of AFL and 500,000 incident cases of AF. ${ }^{12}$ Since AFL and AF frequently coexist, however, we estimate that the number of individuals with their first ever episode of one of these arrhythmias but not the other would be 84,000 incident cases of AFL only and 395,000 incident cases of AF only.

Despite recent therapeutic advances and the fact that both arrhythmias are now potentially ablated, AFL and AF remain independent predictors of major adverse outcomes. A report from MESA showed that even after adjusting for age, sex, and several comorbid conditions, persons diagnosed with either of these atrial tachyarrhythmias in the 1990s have about a 2-fold increased risk of mortality compared to controls in models. ${ }^{7}$

The precise determinants of atrial arrhythmogenesis in humans remain largely unknown. Recent animal studies have provided significant insights into the pathways involved in remodeling, and have indicated the pathophysiological role of remodeling in specific contexts. ${ }^{13}$ As in clinical practice, experimental models have shown that AFL and AF typically occur in the setting of altered structural and electrical substrates. ${ }^{14}$

Our data show the existence of identifiable differences in the etiologic mechanisms that contribute to the onset of these arrhythmias. Our data suggest that while AFL and AF may share certain common risk factors, these two atrial tachyarrhythmias may also differ in the conditions that predispose their development. We found important differences in 4 of the 11 variables pre-selected for analysis. COPD, CHF, and smoking were all more common among incident cases of AFL, while a history of hypertension was more likely to be present in those with AF. We believe these data suggest that the distinct arrhythmogenic substrates required for the initiation and maintenance of these tachyarrhythmias in humans may be partly determined by selective influences of specific predisposing comorbidities.

The role of $\mathrm{CHF}$ in induction of atrial interstitial fibrosis which leads to AF has been documented in multiple animal studies. ${ }^{15-17}$ We postulated that COPD may predispose patients to cardiac arrhythmia either by the direct effects on the right heart through pulmonary hypertension, right ventricular strain, and stretching of the right atrium or as a result of therapeutic modalities used to treat COPD. The effect of metabolic changes (hypoxia and acidosis) could be significant. Multiple studies on human subjects have shown that salbutamol via its beta- 2 action significantly enhances atrioventricular nodal conduction and reduces atrioventricular nodal, atrial, and ventricular refractoriness. ${ }^{18,19}$ Epidemiological studies have shown that patients with COPD and asthma treated with oral steroids were at an increased risk of developing AF even after controlling for a few factors of disease severity. ${ }^{20}$ van der Hooft et $\mathrm{al}^{21}$ also found a higher incidence of AF among patients requiring high dose oral steroids regardless of the etiology (rheumatic, allergic, or malignant hematological diseases). One mechanism by which steroids facilitate arrhythmogenesis is via a direct effect on the cell membrane causing potassium efflux from cells. ${ }^{22}$ Theophylline has also been shown to be associated with AF among patients with COPD, interestingly even with normal serum levels. ${ }^{23}$ Theophylline has been shown to decrease the atrioventricular and sinoatrial conduction time significantly while increasing the serum concentration of epinephrine and nor-epinephrine. ${ }^{24}$ Reduction in the atrial refractory period along with dispersed recovery of the excitability secondary to theophylline is presumed to cause multiple reentrant circuits leading to AF. Our study was not designed to address the confounding effects of medications or severity of illness. More basic research and population-based studies are needed to confirm or refute this epidemiological association with AFL but not with AF to have potential clinical or therapeutic implications.

There could be multiple potential physiologic explanations for our findings. In typical AFL for example, it is now clear that its characteristic macro-reentrant circuit is normally limited to the right atrium. ${ }^{25}$ The flutter circuit travels cranially up the interatrial septum and caudally down through the free wall of the right atrium. The area of slow conduction (isthmus), usually located in the inferior portion of the right atrial chamber, is caused by functional rather than constant block. This zone is flanked by areas of anatomical block caused by the inferior vena cava and the tricuspid valve. ${ }^{26}$ It is certainly conceivable, if not likely, that the electroanatomical substrate required for maintenance of that AFL circuit might be facilitated by the impact of long standing COPD and CHF, as these conditions may all cause increased right atrial pressure, wall stress, and myocardial stretching. 
The most important breakthrough in understanding the pathophysiology of AF was the description of focal discharges arising from the pulmonary veins near the junction where these veins drain into the left atria, which potentially initiate and perpetuate the cycle. ${ }^{1,25}$ We postulate that the association of AF with systematic hypertension could occur from the relatively greater impact on the left atria of the latter.

We minimized selection bias by identifying all new cases of AFL or AF occurring in the entire population in this region and by using the resources of MESA. Given the intermittent nature of these arrhythmias, frequent lack of symptoms, and obvious technologic requirements, their complete ascertainment in any population would require compulsory, long-term continuous monitoring of all individuals in a given area. By limiting the comparative analysis to subjects with only one of these arrhythmias, we excluded $21 \%$ of the incident cases of AF and $58 \%$ of the incident cases of AFL. Similar to other population-based studies, our ability to conduct subanalyses of certain subsets was limited. We were unable to account for severity of illness in COPD, CHF, and hypertension. Despite a total enrollment of 577 incident cases, sample size was a limitation. There are several other comorbid conditions and pathophysiologic states that must be evaluated. Also, echocardiographic parameters were not evaluated. Lastly, the population of MESA is predominantly white and rural.

To our knowledge, this study represents the first investigation to compare the prevalence of comorbidities preceding the onset of AF or AFL in a geographically-defined population. Our findings may have potentially important mechanistic and clinical implications and provide a new potential area for future research into the development and thus prevention of these common arrhythmias.

\section{Acknowledgments}

The authors thank Marshfield Clinic Research Foundation for its support through the assistance of Marie Fleisner and Alice Stargardt in the preparation of this manuscript.

\section{References}

1. Haïssaguerre M, Jaïs P, Shah DC, Takahashi A, Hocini M, Quiniou G, Garrigue S, Le Mouroux A, Le Métayer P, Clémenty J. Spontaneous initiation of atrial fibrillation by ectopic beats originating in the pulmonary veins. N Engl J Med 1998;339:659-666.

2. Khaykin Y, Marrouche NF, Saliba W, Schweikert R, Bash D, Chen MS, Williams-Andrews M, Saad E, Burkhardt DJ, Bhargava M, Joseph G, Rossillo A, Erciyes D, Martin D, Natale A. Pulmonary vein antrum isolation for treatment of atrial fibrillation in patients with valvular heart disease or prior open heart surgery. Heart Rhythm 2004;1:33-39.

3. DeStefano F, Eaker ED, Broste SK, Nordstrom DL, Peissig PL, Vierkant RA, Konitzer KA, Gruber RL, Layde PM. Epidemiologic research in an integrated regional medical care system: the Marshfield Epidemiologic Study Area. J Clin Epidemiol 1996;49:643-652.

4. Greenlee RT. Measuring disease frequency in the Marshfield Epidemiologic Study Area (MESA). Clin Med Res $2003 ; 1: 273-280$
5. Orejarena LA, Vidaillet H Jr, DeStefano F, Nordstrom DL, Vierkant RA, Smith PN, Hayes JJ. Paroxysmal supraventricular tachycardia in the general population. J Am Coll Cardiol 1998;31:150-157.

6. Granada J, Uribe W, Chyou PH, Maassen K, Vierkant R, Smith PN, Hayes J, Eaker E, Vidaillet H. Incidence and predictors of atrial flutter in the general population. J Am Coll Cardiol 2000;36:2242-2246.

7. Vidaillet H, Granada JF, Chyou PH, Maassen K, Ortiz M, Pulido JN, Sharma P, Smith PN, Hayes J. A population-based study of mortality among patients with atrial fibrillation or flutter. Am J Med 2002;113:365-370.

8. Chikani V, Reding D, Gunderson P, McCarty CA. Psychosocial work characteristics predict cardiovascular disease risk factors and health functioning in rural women: the Wisconsin Rural Women's Health Study. J Rural Health 2005; 21:295-302.

9. Sharma PP, Greenlee RT, Anderson KP, Chyou PH, Osorio HJ, Smith PN, Hayes JH, Vidaillet H. Prevalence and mortality of patients with myocardial infarction and reduced left ventricular ejection fraction in a defined community: relation to the second multicenter automatic defibrillator implantation trial. J Interv Card Electrophysiol 2007;19:157-164.

10. Go AS, Hylek EM, Phillips KA, Chang Y, Henault LE, Selby JV, Singer DE. Prevalence of diagnosed atrial fibrillation in adults: national implications for rhythm management and stroke prevention: the AnTicoagulation and Risk Factors in Atrial Fibrillation (ATRIA) Study. JAMA 2001; 285:2370-2375.

11. Fuster V, Rydén LE, Cannom DS, Crijns HJ, Curtis AB, Ellenbogen KA, Halperin JL, Le Heuzey JY, Kay GN, Lowe JE, Olsson SB, Prystowsky EN, Tamargo JL, Wann S, Smith SC Jr, Jacobs AK, Adams CD, Anderson JL, Antman EM, Halperin JL, Hunt SA, Nishimura R, Ornato JP, Page RL, Riegel B, Priori SG, Blanc JJ, Budaj A, Camm AJ, Dean V, Deckers JW, Despres C, Dickstein K, Lekakis J, McGregor K, Metra M, Morais J, Osterspey A, Tamargo JL, Zamorano JL; American College of Cardiology/American Heart Association Task Force on Practice Guidelines; European Society of Cardiology Committee for Practice Guidelines; European Heart Rhythm Association; Heart Rhythm Society. ACC/AHA/ESC 2006 Guidelines for the Management of Patients with Atrial Fibrillation: a report of the American College of Cardiology/American Heart Association Task Force on Practice Guidelines and the European Society of Cardiology Committee for Practice Guidelines (Writing Committee to Revise the 2001 Guidelines for the Management of Patients With Atrial Fibrillation): developed in collaboration with the European Heart Rhythm Association and the Heart Rhythm Society. Circulation 2006; 114:e257-e354.

12. Greenlee RT, Vidaillet H. Recent progress in the epidemiology of atrial fibrillation. Curr Opin Cardiol 2005;20:7-14.

13. Kistler PM, Sanders P, Dodic M, Spence SJ, Samuel CS, Zhao C, Charles JA, Edwards GA, Kalman JM. Atrial electrical and structural abnormalities in an ovine model of chronic blood pressure elevation after prenatal corticosteroid exposure: implications for development of atrial fibrillation. Eur Heart J 2006;27:3045-3056.

14. Allessie MA, Boyden PA, Camm AJ, Kléber AG, Lab MJ, Legato MJ, Rosen MR, Schwartz PJ, Spooner PM, Van Wagoner DR, Waldo AL. Pathophysiology and prevention of atrial fibrillation. Circulation 2001;103:769-777.

15. Boixel C, Fontaine V, Rücker-Martin C, Milliez P, Louedec L, Michel JB, Jacob MP, Hatem SN. Fibrosis of the left atria during progression of heart failure is associated with increased matrix metalloproteinases in the rat. J Am Coll Cardiol 2003;42:336-344. 
16. Li D, Fareh S, Leung TK, Nattel S. Promotion of atrial fibrillation by heart failure in dogs: atrial remodeling of a different sort. Circulation 1999;100:87-95.

17. Nattel S. Defining "culprit mechanisms" in arrhythmogenic cardiac remodeling. Circ Res 2004;94:1403-1405.

18. Insulander P, Juhlin-Dannfelt A, Freyschuss U, Vallin H. Electrophysiologic effects of salbutamol, a beta2-selective agonist. J Cardiovasc Electrophysiol 2004;15:316-322.

19. Kallergis EM, Manios EG, Kanoupakis EM, Schiza SE, Mavrakis HE, Klapsinos NK, Vardas PE. Acute electrophysiologic effects of inhaled salbutamol in humans. Chest 2005; 127:2057-2063.

20. Huerta C, Lanes SF, Garcia Rodriguez LA. Respiratory medications and the risk of cardiac arrhythmias. Epidemiology 2005;16:360-366.

21. van der Hooft CS, Heeringa J, Brusselle GG, Hofman A, Witteman JC, Kingma JH, Sturkenboom MC, Stricker BH. Corticosteroids and the risk of atrial fibrillation. Arch Intern Med 2006;166:1016-1020.

22. Fujimoto S, Kondoh H, Yamamoto Y, Hisanaga S, Tanaka K. Holter electrocardiogram monitoring in nephrotic patients during methylprednisolone pulse therapy. Am J Nephrol 1990;10:231-236.

23. Varriale P, Ramaprasad S. Aminophylline induced atrial fibrillation. Aminophylline induced atrial fibrillation. Pacing Clin Electrophysiol 1993;16:1953-1955.

24. Eiriksson CE Jr, Writer SL, Vestal RE. Theophylline-induced alterations in cardiac electrophysiology in patients with chronic obstructive pulmonary disease. Am Rev Respir Dis 1987; 135:322-326.

25. Cosío FG, López Gil M, Arribas F, Palacios J, Goicolea A, Núñez A. Mechanisms of entrainment of human common flutter studied with multiple endocardial recordings. Circulation 1994;89:2117-2125.

26. Waldo AL. Mechanisms of atrial flutter and atrial fibrillation: distinct entities or two sides of a coin? Cardiovasc Res 2002;54:217-229.

\section{Author Affiliations}

Ravi K. Mareedu, MD*; Ihab B. Abdalrahman, MD*; Kodlipet C. Dharmashankar, MD*; Juan F. Granada, ${ }^{*}$; Po-Huang Chyou, PhD ; Param P. Sharma, MD\$; Peter N. Smith, MD\$; John J. Hayes, MD\$; Robert T. Greenlee, PhD"; Humberto Vidaillet, $M D^{\S}$

Departments of *Internal Medicine and §Cardiology, Marshfield Clinic and St. Joseph's Hospital, Marshfield, WI; †Skirball Center for Cardiovascular Research, Cardiovascular Research Foundation, New York, NY; $¥$ Biomedical Informatics Research Center, and \#Epidemiology Research Center, Marshfield Clinic Research Foundation, Marshfield, WI 